\title{
Viscous State Effect on the Activity of Fe Nanocatalysts
}

\author{
Felipe Cervantes-Sodi, ${ }^{\dagger}$ Thomas P. McNicholas, ${ }^{\ddagger}$ Jay G. Simmons, Jr, ${ }^{\ddagger}$ Jie Liu, ${ }^{\star}$ Gabor Csányi, ${ }^{\dagger}$ \\ Andrea C. Ferrari, ${ }^{\dagger}$ and Stefano Curtarolo ${ }^{\S, \perp, *}$ \\ ${ }^{\dagger}$ Department of Engineering, University of Cambridge, Cambridge, U.K. and ${ }^{\ddagger}$ Department of Chemistry, ${ }^{\S}$ Department of Mechanical Engineering and Materials Science, and \\ ${ }^{\perp}$ Department of Physics, Duke University, Durham, NC, United States
}

ABSTRACT Many applications of nanotubes and nanowires require controlled bottom-up engineering of these nanostructures. In catalytic chemical vapor deposition, the thermo-kinetic state of the nanocatalysts near the melting point is one of the factors ruling the morphology of the grown structures. We present theoretical and experimental evidence of a viscous state for nanoparticles near their melting point. The state exists over a temperature range scaling inversely with the catalyst size, resulting in enhanced self-diffusion and fluidity across the solid-liquid transformation. The overall effect of this phenomenon on the growth of nanotubes is that, for a given temperature, smaller nanoparticles have a larger reaction rate than larger catalysts.

KEYWORDS: nanocatalysis · thermodynamics $\cdot$ kinetics $\cdot$ growth rate

*Address correspondence to stefano@duke.edu.

Received for review August 2, 2010 and accepted October 5, 2010.

Published online October 21, 2010. $10.1021 / \mathrm{nn} 101883 \mathrm{~s}$

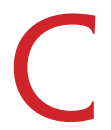

atalytic chemical vapor deposition (CVD) is an effective site/sizeselective synthesis technique. ${ }^{1-7}$ The properties of the catalytic particle play a key role in the nucleation and growth processes, as well as in the final nanostructure morphology. ${ }^{7-11}$ At the nanoscale, two aspects are inter-related: the possibility of bulk and surface diffusion of the feedstock in and on the nanoparticles ${ }^{12-14}$ and the thermodynamic state of the catalyst interacting with the growing nanostructure. ${ }^{12-16}$ Feedstock diffusion has been thoroughly investigated in previous works, ${ }^{12-14}$ but the thermodynamic issue has not been explored in its full complexity.

At the nanoscale, the macroscopic dichotomy liquid/solid is not well-defined, and the issue of size-induced viscosity $\eta$ arises. This affects the catalyst activity by changing its melting point, ${ }^{15,16}$ solubility, ${ }^{17,18}$ and tendency to coarsen. ${ }^{19}$ Kinetic properties, such as diffusion coefficients ${ }^{20}$ and viscosity, can be used to study the particle state as a function of temperature and size. These quantities, intrinsically related to the time frame of the underlying phenomenon, can be extracted by monitoring an out-ofequilibrium effect defining rate of events.
Thus, for a fixed rate of events, the long- or short-time frames of the system can be inferred by the inverse of the monitored value. Legitimate observables can be selected among diffusion, ${ }^{20}$ viscoelasticity, and thermal conductivity coefficients. Within this approach, it is possible to describe phenomena like dynamic coexistence ${ }^{19,21-23}$ in systems having short-order crystallinity and viscous response to external forces, such as the stress generated by a growing nanostructure. For example, nanocatalysts can change shape as a result of interactions with the carbon nanotubes (CNT) walls. ${ }^{4}$ This can be described by the time fluctuations of a statistical-bondlength order parameter ${ }^{16}$ known as the Lindemann index, $\delta .^{24}$

Here, we analyze the nanoparticles near the melting point by investigating, as functions of size, the melting depression and the self-diffusion coefficient $D$ leading to viscosity $(\eta \propto 1 / D)$.

\section{RESULTS AND DISCUSSION}

In nanoparticles, the melting point is inversely proportional to the diameter through the Gibbs - Thomson (GT) relation. ${ }^{9,19,21,22}$ We describe this by using classical molecular dynamics (MD) (see Methods for details). We characterize the melting by the change in internal energy, with associated latent heat, and by the variation in the Lindemann index statisticalbond-length order parameter, $\delta$, with respect to the temperature $T$. Fick's law ${ }^{25}$ describes the exponential behavior of the bulk diffusion as a function of $T$. For bulk iron at the melting temperature $T_{\mathrm{m}}, D\left(T_{\mathrm{m}}\right) \approx 4.16 \times$ $10^{-5} \mathrm{~cm}^{2} / \mathrm{s}^{26-29}$ This measures the diffusion at the formation of the liquid state. When size decreases to the nanometer scale, one might expect a variation on $D\left(T_{\mathrm{m}}\right)$ be- 

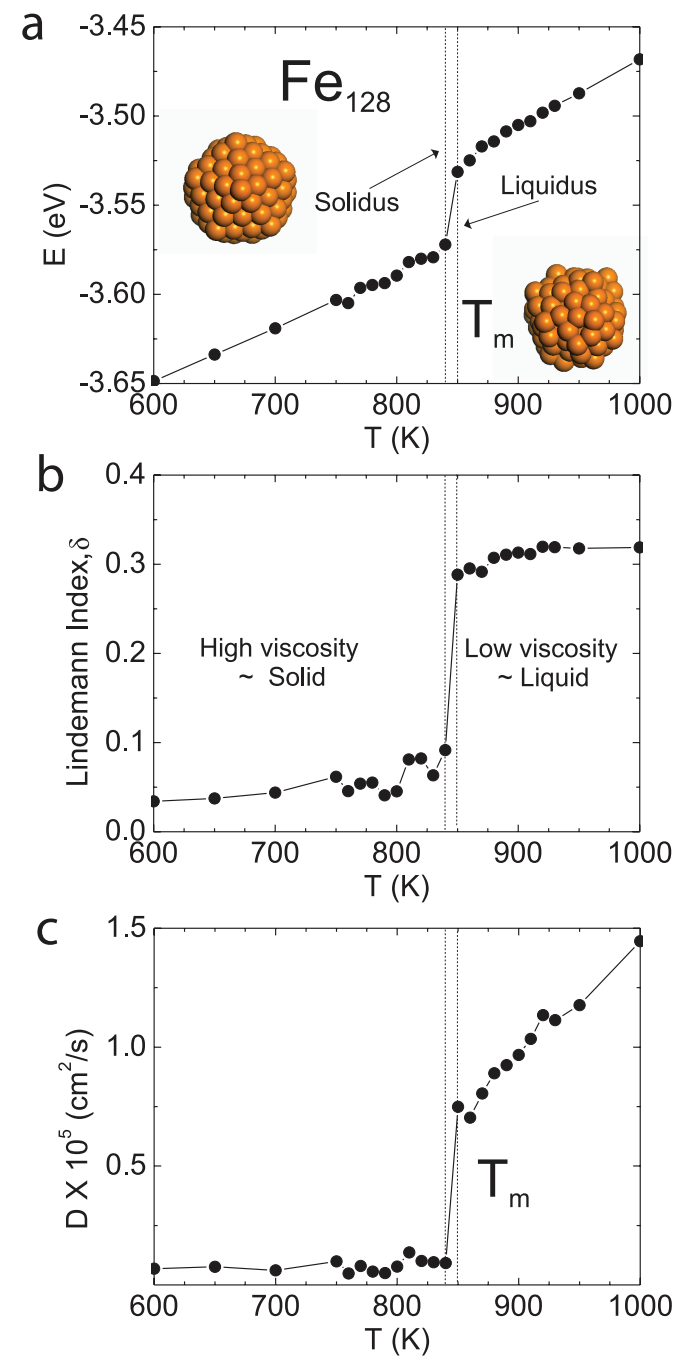

Figure 1. (a) Total energy. Specific heats in the liquid and solid phases; (b) Lindemann index, $\delta$, and sudden variation in the long-range order on the cluster; (c) diffusion coefficient with different behavior in the liquid and solid phases.

cause of the GT melting depression. ${ }^{15,30-32}$ Using MD, we monitor $\delta$, starting from below $T_{\mathrm{m}}$ (estimated from the GT relation ${ }^{15}$ ), while applying heat in small increments to drive the system across the phase transition. High/low values of $\delta$ identify the liquid/solid state, respectively. Concurrently, we monitor the self-diffusion coefficient calculated within the Green-Kubo formalism. ${ }^{56}$

We limit our study to pure Fe particles for three reasons. First, it has been shown with ab initio molecular dynamics that pure Fe nanoparticles can grow CNTs. ${ }^{33}$ Second, active Fe particles equilibrate into a solid solution $\mathrm{Fe}-\mathrm{C}$ mixture, ${ }^{17}$ as nucleation of ordered $\mathrm{Fe}_{3} \mathrm{C}$ can be detrimental for growth. ${ }^{16}$ (This argument has also been extended to $\mathrm{Fe}-\mathrm{Mo}-\mathrm{C}$ mixtures in ref 34). Third, with solubility of $C$ in Fe reducing with decreasing particle size, ${ }^{17}$ the main effect of solute $C$ is to further reduce the melting point $\left(\mathrm{Fe}-\mathrm{C}\right.$ liquidus $\left.{ }^{35}\right)$, unless the particle size becomes www.acsnano.org
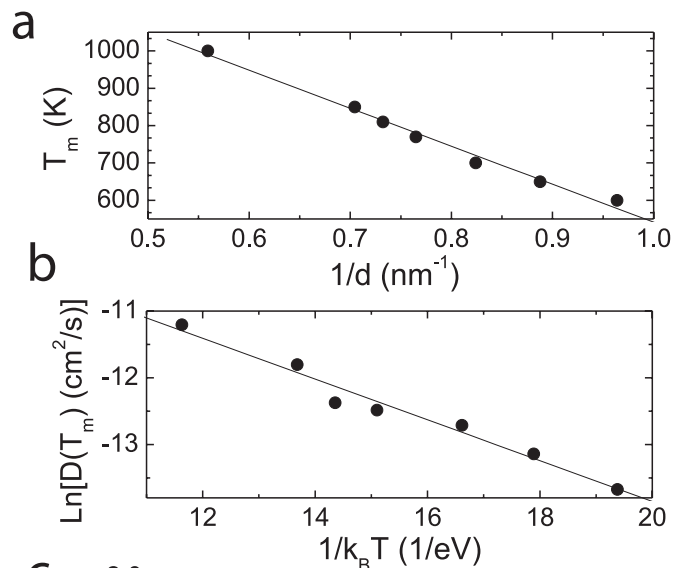

C

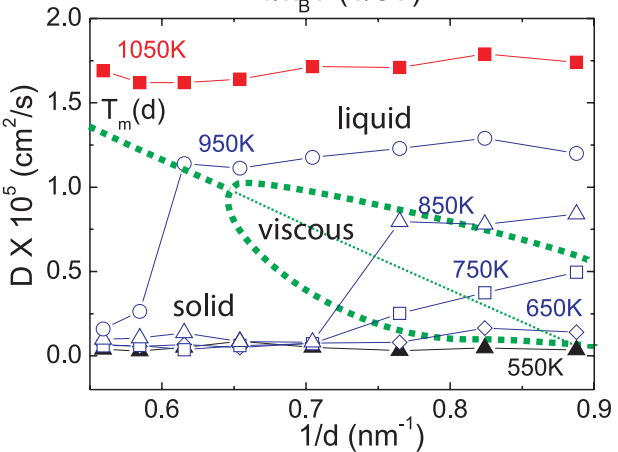

Figure 2. (a) Maximum solid point $T$ (liquidus), $T_{\mathrm{m}}$, as a function of diameter. (b) Arrhenius behavior of the diffusion coefficient at the melting point. Linear fits are shown. (c) Diffusion coefficient as a function of diameter at six different $T$. The viscous region is defined as the range of sizes and $T$ in which the diffusion coefficients is between solid and liquid values and grows continuously.

too small to promote nucleation of $\mathrm{Fe}_{3} \mathrm{C}$, (ref 36 ), destabilizing the CNT growth. ${ }^{17}$

A $\mathrm{Fe}_{128}$ cluster is our first case study. We consider $T$ increasing from 600 to $1000 \mathrm{~K}$, the typical range for high quality CNT growth. ${ }^{1-4}$ Figure 1 a shows the caloric curve $E(T)$ (energy per atom as a function of $T$ ). The discontinuity in $E(T)$ equals the latent heat $\Delta H$. From the caloric curve we get $T_{\mathrm{m}}=850 \mathrm{~K}$. Figure $1 \mathrm{~b}$ depicts $\delta$ for the same particle. After an oscillating behavior of the precursors at $T \approx 800-840 \mathrm{~K}$, long-range disorder is reached at $T=850 \mathrm{~K}$. Owing to the finite size of the particle, melting in a single-species cluster is not a zerodimensional invariant point, unlike in the case of bulk materials, ${ }^{15,19}$ as it occurs in a range $\Delta T_{\mathrm{m}}$. In our case $\Delta T_{\mathrm{m}}$ is $840-850 \mathrm{~K}$, where the solid and liquid phases co-

\begin{tabular}{|c|c|}
\hline $\begin{array}{l}\text { average Fe nanoparticle, } \\
\text { diameter }(\mathrm{nm})\end{array}$ & $\begin{array}{c}\text { average carbon nanotube, } \\
\text { length }(\mu \mathrm{m})\end{array}$ \\
\hline 1.2 & 1083 \\
\hline 2 & 813 \\
\hline 3 & 639 \\
\hline
\end{tabular}

${ }^{a}$ Average SWNTs length, $L_{i}$, produced from nanoparticles with increasing diameters, $2 R_{i}$, over a $\Delta t=15$ minutes growth time. The average SWNTs length decreases with increasing nanoparticle diameter. 

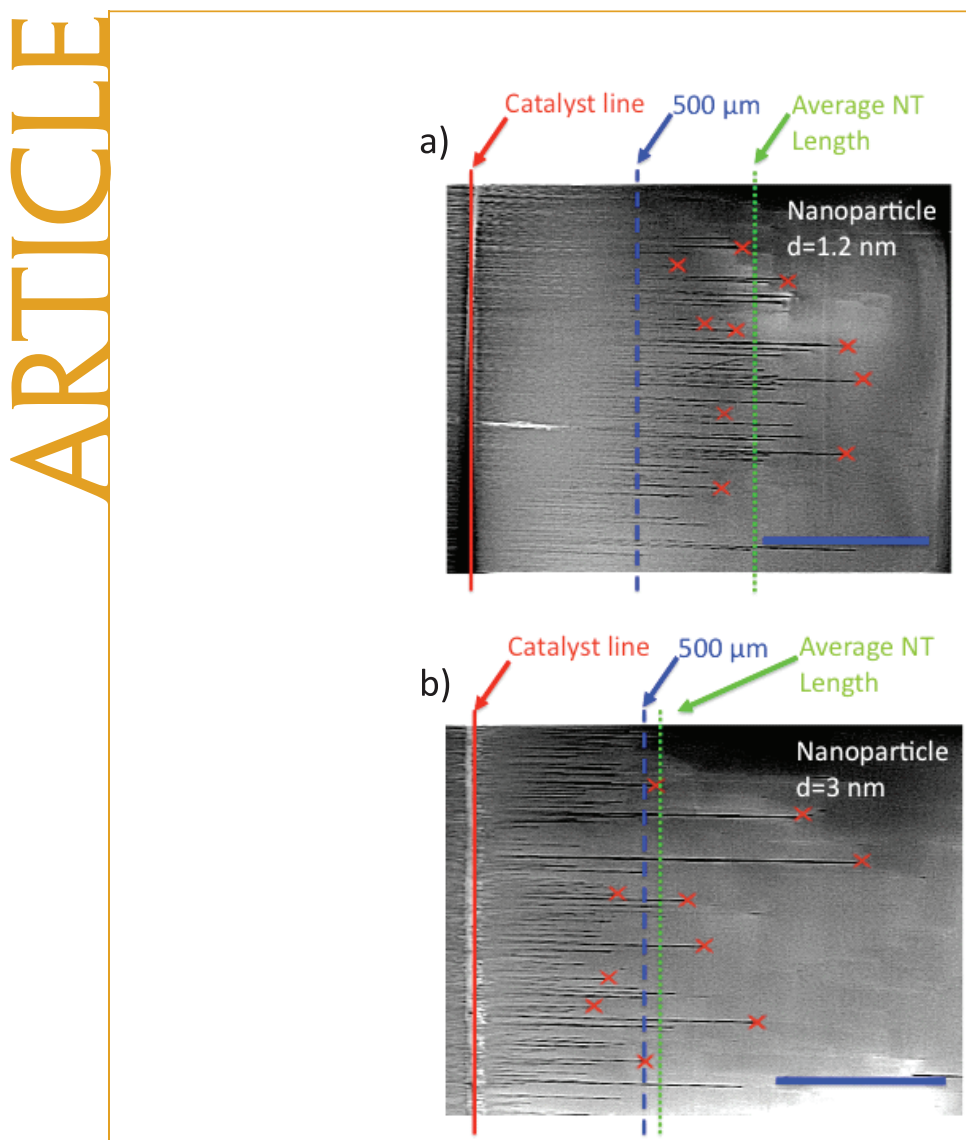

Figure 3. SWNTs grown from metal nanoparticles with different average diameter: (a) $1.2 \mathrm{~nm}$; (b) $3 \mathrm{~nm}$. Red vertical lines indicate the original position of the metal particles. Crosses highlight the end length of some representative tubes. Green vertical lines indicate the average CNT length as derived from SEM from at least 30 CNTs. Blue vertical lines mark $500 \mu \mathrm{m}$ from the original position of the metal particles.

exist in different fractions and time frames (dynamic coexistence $^{15,16,22,31,32,37,38}$ ). We thus introduce two definitions: $T_{\mathrm{f}}$, the minimum-liquid point (solidus), below which the particle is only solid, ${ }^{15}$ and $T_{\mathrm{m}}$, the maximum-solid point (liquidus), above which the particle is only liquid. ${ }^{15}$ Between $T_{\mathrm{f}}$ and $T_{\mathrm{m}}$ the nanoparticle fluctuates between the solid and liquid states $32,37,38$ and overall resembles a viscous droplet of atoms. Figure 1c plots the diffusion coefficient. At $T$ below $T_{\mathrm{f}} D$ is similar to the bulk solid $\left(<1.5 \times 10^{-7} \mathrm{~cm}^{2} / \mathrm{s}^{39}\right)$. At

a)

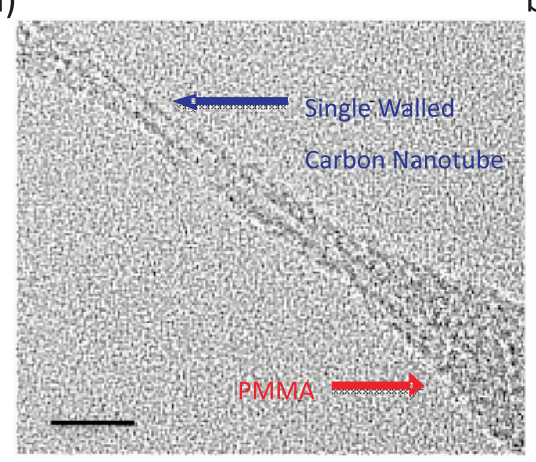

b)

$T_{\mathrm{m}}=850 \mathrm{~K}, D$ drastically increases to $D\left(T_{\mathrm{m}}\right)=7.5 \times$ $10^{-6} \mathrm{~cm}^{2} / \mathrm{s}$. This is larger than $D$ in the bulk solid and what we find in solid nanoparticles $\left(<10^{-7} \mathrm{~cm}^{2} / \mathrm{s}\right)$, but much smaller than $D$ at the bulk melting point in the liquid phase $\left(4.16 \times 10^{-5} \mathrm{~cm}^{2} / \mathrm{s}^{26,27}\right)$, and similar to previous reports of self-diffusion in molten $\mathrm{Fe}_{331}: 6.17 \times 10^{-6} \mathrm{~cm}^{2} / \mathrm{s} .{ }^{40}$ Although the molten nanoparticle has a $D\left(T_{\mathrm{m}}\right)$ between bulk solid and liquid $\left(4.16 \times 10^{-5} \mathrm{~cm}^{2} / \mathrm{s}^{26,27}\right)$, the $T$ range of dynamic coexistence enhances this diffusion, Figure 2.

Figure 2a shows $T_{\mathrm{m}}$ of $\mathrm{Fe}_{\mathrm{N}}$ for $N=50-256(d \approx$ $1-1.7 \mathrm{~nm}) . T_{\mathrm{m}}$ is estimated from the caloric curve, Lindemann index, and diffusion coefficient, depending on the particle diameter. ${ }^{15}$ These results, consistent with previous studies of $\mathrm{Fe}$ and other metals, ${ }^{15,30,32}$ are used to correlate with $D\left(T_{\mathrm{m}}\right)$. The Arrhenius behavior of $D\left(T_{\mathrm{m}}\right)$ it is conserved at the nanoscale (Figure $2 \mathrm{~b}$ ), with $E_{\mathrm{a}}=0.305 \mathrm{eV}, D_{0}=4.33$ $\times 10^{-4} \mathrm{~cm}^{2} / \mathrm{s}$. The extrapolation of $T_{\mathrm{m}}$ for bulk Fe $\approx$ $1558 \pm 40 \mathrm{~K}$ underestimates the experimental value by $\sim 14 \%$. The values of $D$ at $T_{\mathrm{m}}$ with these $E_{\mathrm{a}}$ and $D_{0}$ are within an order of magnitude of those reported by refs 26 and 40 for the case of infinite $\mathrm{Fe}$ $\left(\sim 10^{-5} \mathrm{~cm}^{2} / \mathrm{s}\right)$ and a cluster of $\mathrm{Fe}_{331}\left(\sim 10^{-6} \mathrm{~cm}^{2} / \mathrm{s}\right)$ at the appropriate $T_{\mathrm{m}}$. Our $D$ values are slightly higher also because the higher surface to volume ratio in the nanoparticles gives a large surface diffusion contribution to the average total $D$. The calculations consider only floating particles, as the effect of the substrate is to reduce the GT melting depression as a function of particle/substrate interaction, ${ }^{41}$ thereby shifting the curves and regions of Figure 2 toward higher $T$, without necessarily modifying their features.

Figure 2 c depicts $D$ as a function of diameter for a set of $T$ and illustrates the size-induced enhanced diffusion. There are three scenarios. (1) Liquid. When the particle is liquid, diffusion follows the Arrhenius law dependence of Figure $2 \mathrm{~b}$ and is sizeindependent. (2) Solid. In this case (low- $T$ or largesize), the particle is solid and $D$ is both $T$ - and sizeindependent. (3) Viscous. Size-induced enhanced diffusion is contained within the region: the particle

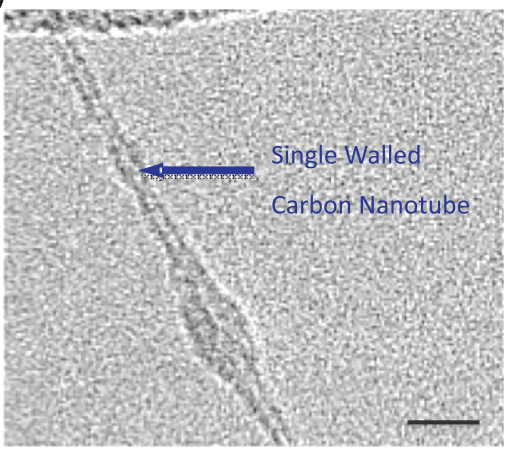

Figure 4. TEM images of nanotubes-grown $3 \mathrm{~nm}$ catalysts, showing they are single wall. Some residual PMMA, poly(methylmethacrylate), remains on the tubes after transferring onto TEM grids. (Scale bars: $10 \mathrm{~nm}$ ). 
can be seen as solid or liquid, depending on the observation time frame. Viscosity is driven by dynamic coexistence. ${ }^{15,22}$ For example, in the ranges of Figure $2 \mathrm{c}(T=650-950 \mathrm{~K}, d=1.1-1.8 \mathrm{~nm})$, the clusters are viscous even well below the melting point (e.g., the points at $650-850 \mathrm{~K}$ on the left of $T_{\mathrm{m}}$ ). Furthermore, $D$ becomes larger as the nanoparticle size becomes smaller.

Isothermally, size-induced enhanced diffusion might have important consequences for the catalytic growth of nanostructures. We infer the following. During CNT growth at constant $T$ with a distribution of Fe catalysts with $d \geq 1 \mathrm{~nm}$, smaller particles are more fluid and their enhanced self-diffusion allows a more rapid surface reorganization, promoting the carbon feedstock transformation. Thus, we expect small clusters to produce CNTs at a faster rate than larger ones. However, if the catalyst size becomes too small, surface-tension dominates over bulk and surface contributions to the free-energy, causing reduced solubility, detrimental for catalytic activity due to nucleation of non active $\mathrm{Fe}_{3} \mathrm{C}^{18}$ If diffusion in the viscous region is enhanced, without deactivation, the stress field exerted by the growing CNT will reshape the catalyst.

To validate the above suggestions, we perform two sets of experiments. First, single wall carbon nanotubes (SWNTs) are grown on quartz using variously sized Fe nanoparticles. In order to favor directional growth, thus easier evaluation and comparison of CNT lengths, we use quartz as a sustrate. ${ }^{42}$ The growth conditions are the same as for refs 43 and 44 (see Methods for details). The particles are characterized by atomic force microscopy (AFM) and transmission electron microscopy (TEM). Growth is carried out under identical conditions for all catalysts diameters. Scanning electron microscopy (SEM) is used to measure their length (Table 1). We find that smaller diameter nanoparticles result in longer SWNTs (see Figure 3). TEM confirms that the CNTs are SWNTs, as illustrated in Figure 4.

A dimensional analysis can be performed with the values of Table 1. Let us consider steady state growth of nanotubes. If the dissociation rate is proportional to the surface area of the catalyst ( $\beta R^{2}$, where $\beta$ is a processdependent proportionality constant and $R$ is the radius of the particle), and the mobile carbon atoms are required to drift with a time-constant $\tau$ a distance of the same order of magnitude of the particle (through surface-, subsurface- or bulk-diffusion), then we obtain that the rate of available carbon atoms for growing nanotubes is $\beta R^{2}(R / \tau)$. For a given growth time $\Delta t, 2 \pi R L \rho$ atoms are needed to grow a CNT of length $L$ and $\rho$ carbon atoms suface density. Thus, for conservation of mass, we have $\beta R^{2}(R / \tau) \Delta t=2 \pi R L \rho$. The comparison of two different catalysts' sizes $(i, j)$ within the same process $(\beta, \rho$ are constant, $\Delta t$ is chosen, and $R, L$ are observable) leads to $\tau_{i} L_{i} / R_{i}^{2}$ $=\tau_{j} L_{j} / R_{j}^{2}$ and finally to $\tau_{i} / \tau_{j}=\left(R_{i}^{2} / L_{i}\right)\left(L_{j} / R_{j}^{2}\right)$. For example, by substituting the values of Table 1 , we obtain $\tau_{1} / \tau_{2}=$ 0.27 and $\tau_{2} / \tau_{3}=0.35$. The time-constant ratios indicate a)
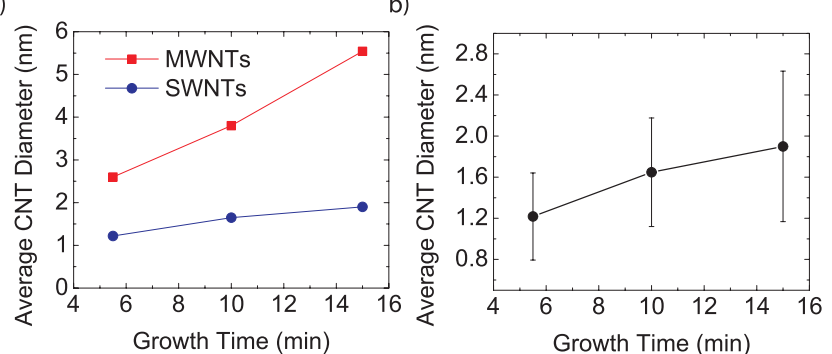

Figure 5. (a) Average CNT diameter as a function of growth time for nanoparticles with (blue) $1-2 \mathrm{~nm}$ and (red) 2.5-7.6 nm diameters. (b) SWNT radius and standard deviation. The two quantities increase with time.

that the smaller is the catalyst, the higher is the mobility required for a given supply of carbon atoms to satisfy overall mass conservation.

Besides the catalyst particle size, other factors may prevent CNTs from growing very long. Accordingly, an additional experiment was designed to confirm the values in Table 1. Here, the time-dependence of the CNT diameter distribution is characterized. Using two catalysts diameter distributions ( $\sim 1-2 \mathrm{~nm}$ and $\sim 2.5-7.6 \mathrm{~nm}$ ), three growths are conducted under identical conditions for $5.5,10$, and $15 \mathrm{~min}$. Since larger particles nucleate and grow CNTs slower than smaller catalysts, their observable contribution to the CNT arrays is expected to appear more slowly. Therefore, the average CNT diameter should increase with growth time.

Indeed, Figure 5a shows that the average CNT diameter increases with increasing time, indicating that smaller diameter nanoparticles nucleated first and produce CNTs before larger ones. This happens both in the nanoparticle size regime corresponding to SWNTs as well as MWNTs.

This also agrees with the theoretical findings that smaller particles must be more fluid and prone to adapt and diffuse other species.

Furthermore, the diameter standard deviation also increases with time, Figure 5b. This agrees well with theory: the smaller diameter, and therefore more fluid, nanoparticles are predicted to nucleate and grow tubes before larger diameter ones. Thus, as growth time increases, a larger distribution of nanotube diameters is expected. ${ }^{45}$ This distribution broadens to include progressively larger diameter nanotubes within the limits of the catalyst nanoparticles' diameter distribution. Ultimately, this manifests

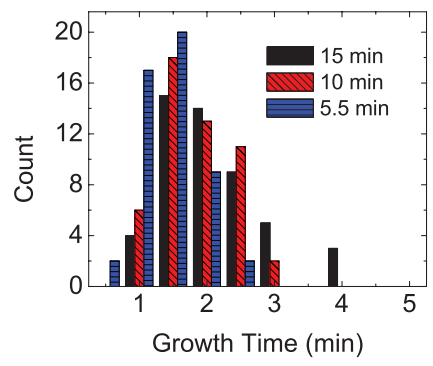

Figure 6. Diameter distributions of samples grown under the same conditions for increasing time.

VOL. 4 - NO. 11 - 6950-6956 - 2010 
itself by increasing the average diameter, as well as its standard deviation, with increasing time. These points are further illustrated in the histograms in Figure 6.

\section{CONCLUSIONS}

We identified, with a combination of thermokinetic theory and growth experiments, a viscous state of nanoparticles near their melting point. This state exists over a temperature range scaling inversely with the nanoparticle size. The enhanced self-diffusion and the consequent fluidity allow a smaller catalyst nanoparticle to better adapt to external conditions and to transform feedstock at a faster rate than larger catalysts.

\section{METHODS}

Molecular Dynamics. Classical molecular dynamics simulations are carried out in a canonical ensemble using the Verlet algorithm ${ }^{46}$ with a time step $t=1 \mathrm{fs}$ and Nose-Hoover thermostat. ${ }^{47}$ We build small Fe nanoparticles with $N<256$, corresponding to $d$ $\approx 1-1.8 \mathrm{~nm}$, relevant for the most common SWNT diameter distribution. For simplicity, we consider floating particles. The substrate-Fe nanoparticle interaction was shown to change the melting depression, ${ }^{15,48}$ which can be parametrized in the GT equation through an effective radius. ${ }^{41,49}$ The $\mathrm{Fe}-\mathrm{Fe}$ interactions are described as a sum of a Born-Mayer-type repulsive and manybody attractive energy terms, ${ }^{50}$ where the coefficients are obtained by fitting the cohesive energy, lattice parameter, and elastic constant of $\gamma-\mathrm{Fe}^{51}$ The potential landscape was shown to be adequate to reproduce CNT growth, ${ }^{52}$ the melting depression, and the $\mathrm{Fe}-\mathrm{C}$ eutectic point. ${ }^{15}$ For the initial particle configuration we perform simulated annealing, to reach stable minima. ${ }^{15}$ Gathering of energies and other averages is performed over $20 \times 10^{6} \mathrm{MD}$ steps.

The statistical-bond-length order parameter Lindemann index is defined as ${ }^{24,53}$

$$
\delta=\frac{2}{N(N-1)} \sum_{i<j} \frac{\sqrt{\left\langle r_{i j}^{2}\right\rangle-\left\langle r_{i j}\right\rangle^{2}}}{\left\langle r_{i j}\right\rangle}
$$

where $r_{i j}$ is the distance between atoms $i$ and $j, N$ the number of particles, and the average is calculated over an MD run at a given $T$. We identify melting when the index is $>0.25 .{ }^{15}$ Extrapolating for $d \rightarrow \infty$ we estimate $T_{\mathrm{m}}$ for bulk Fe $\approx 1558 \pm 40 \mathrm{~K}$, underestimating the experimental value by $\sim 14 \%{ }^{54}$ The diffusion coefficient is defined as ${ }^{5,56}$

$$
D=\frac{1}{3 N} \int_{0}^{\infty}\left\langle\sum_{j=1}^{N} v_{j}(t) v_{j}(\tau)\right\rangle d t
$$

where $v_{j}(t)$ is the velocity of atom $j$ at time $t$. We initialize $v_{j}(t)$ at every time step, as in ref 56 .

In analogy to previous studies of for solid-liquid transition in small argon clusters, ${ }^{57,58}$ here we use the change in $D$ as indication of phase transition in metallic nanoparticles.

Experimental Section. Two sets of growth experiments are conducted. In the first, we grow CNTs using different-sized Fe nanoparticles. Preparation of the catalysts is accomplished by using the same amount of PVP (polyvinylpyrrolidone) $(0.006 \mathrm{~g}, \mathrm{MW}=$ $55 \mathrm{k}$ ) and $\mathrm{EtOH}\left(15 \mathrm{~mL}\right.$ ) but with different $\mathrm{FeCl}_{3}$ concentrations, as outlined in refs 43 and 44 . Growth is then carried out under identical conditions for all nanoparticles, as for refs 43 and 44 , using $\mathrm{EtOH} / \mathrm{MeOH}(150 \mathrm{sccm} / 300 \mathrm{sccm})$ and $\mathrm{H}_{2}(450 \mathrm{sccm})$ on quartz substrates ${ }^{43,44}$ at $750{ }^{\circ} \mathrm{C}$ for 10 min under flowing $\mathrm{H}_{2}$ followed by $\mathrm{EtOH} / \mathrm{MeOH} / \mathrm{H}_{2}$ at $900{ }^{\circ} \mathrm{C}$ for $15 \mathrm{~min}$. The tube lengths are measured using SEM and averaged from at least 30 samples. Nanoparticle diameters are measured using tapping mode AFM after spin coating quartz wafers at $2000 \mathrm{rpm}$ for $45 \mathrm{~s}$ and removing the PVP by $\mathrm{O}_{2}$ plasma treatment for $15 \mathrm{~min}$. Diameters are averaged from at least 50 nanoparticles over a minimum of three different samples for each size distribution.

The second batch of experiments is conducted using two Fe catalysts: one to produce tubes with diameters between 1 and $2 \mathrm{~nm}$, the other to achieve diameters between 2.5 and 7.6 $\mathrm{nm}$. The first is used to measure the average SWNT diameter as a function of growth time. The second for the same measurements on MWNTs. Growth is conducted as for refs 43 and 44, with the growth time varied from 5.5 to $15 \mathrm{~min}$. Following growth, the diameter distribution is analyzed using tapping mode AFM. Care is taken to tune the amplitude of the cantilever oscillation to ensure negligible nanotube depression from the cantilever tapping. Data are taken from at least 50 tubes over a minimum of three different samples for each growth time.

Acknowledgment. Calculations were performed at the Cambridge HPCF, at the University of loannina RCSS, and the Teragrid Partnership (TACC, Grant No. MCA-07S005). We thank N. Awasthi, A. Kolmogorov, W. Setyawan, and S. Hofmann for useful discussions. F.C.S. acknowledges funding from CONACYT Mexico. A.C.F. acknowledges funding from The Royal Society and ERC Grant NANOPOTS and EPSRC Grant EP/G030480/1. S.C. acknowledges funding from ONR (Nos. N00014-07-1-0878, N00014-07-1-1085, N00014-09-1-0921) and NSF (DMR-0639822) and from the Feinberg Fellowship, Weizmann Institute of Science. T.M., J.S. and J.L. acknowledge support from DOE (DE-FC3605GO15103). F.C. is currently at Departamento de Física y Matemáticas, Universidad Iberoamericana, México.

\section{REFERENCES AND NOTES}

1. Givargizov, E. I. Fundamental Aspects of VLS Growth. J. Cryst. Growth 1975, 31, 20-30.

2. Chhowalla, M.; Teo, K. B. K.; Ducati, C.; Rupesinghe, N. L.; Amaratunga, G. A. J.; Ferrari, A. C.; Roy, D.; Robertson, J.; Milne, W. I. Growth Process Conditions of Vertically Aligned Carbon Nanotubes Using Plasma Enhanced Chemical Vapor Deposition. J. Appl. Phys. 2001, 90, 5308-5317.

3. Fan, S.; Chapline, M. G.; Franklin, N. R.; Tombler, T. W.; Cassell, A. M.; Dai, H. Self-Oriented Regular Arrays of Carbon Nanotubes and Their Field Emission Properties. Science 1999, 283, 512-514.

4. Hofmann, S.; Sharma, R.; Ducati, C.; Du, G.; Mattevi, C.; Cepek, C.; Cantoro, M.; Pisana, S.; Parvez, A.; CervantesSodi, F.; et al. In Situ Observations of Catalyst Dynamics during Surface-Bound Carbon Nanotube Nucleation. Nano Lett. 2007, 7, 602-608.

5. Ren, Z. F.; Huang, Z. P.; Xu, J. W.; Wang, J. H.; Bush, P.; Siegal, M. P.; Provencio, P. N. Synthesis of Large Arrays of Well-Aligned Carbon Nanotubes on Glass. Science 1998, 282, 1105-1107.

6. Cassell, A. M.; Raymakers, J. A.; Kong, J.; Dai, H. J. Large Scale CVD Synthesis of Single-Walled Carbon Nanotubes. J. Phys. Chem. B 1999, 103, 6484-6492.

7. Hofmann, S.; Ducati, C.; Neill, R.; Piscanec, S.; Ferrari, A. C.; Geng, J.; Dunin-Borkowski, R. E.; Robertson, J. Gold Catalyzed Growth of Silicon Nanowires by Plasma Enhanced Chemical Vapor Deposition. J. Appl. Phys. 2003, 94, 6005-6012.

8. Shibuta, Y.; Maruyama, S. Molecular Dynamics Simulation of Formation Process of Single-Walled Carbon Nanotubes by CCVD Method. Chem. Phys. Lett. 2003, 382, 381-386.

9. Li, Y.; Kim, W.; Zhang, Y.; Rolandi, M.; Wang, D.; Dai, H. Growth of Single-Walled Carbon Nanotubes from Discrete Catalytic Nanoparticles of Various Sizes. J. Phys. Chem. B 2001, 105, 11424-11431. 
10. Bandow, S.; Asaka, S.; Saito, Y.; Rao, A.; Grigorian, L.; Richter, E.; Eklund, P. Effect of the Growth Temperature on the Diameter Distribution and Chirality of Single-Wall Carbon Nanotubes. Phys. Rev. Lett. 1998, 80, 3779-3782.

11. Kataura, H.; Kumazawa, Y.; Maniwa, Y.; Ohtsuka, Y.; Sen, R.; Suzuki, S.; Achiba, Y. Diameter Control of Single-Walled Carbon Nanotubes. Carbon 2000, 38, 1691-1697.

12. Hofmann, S.; Csányi, G.; Ferrari, A. C.; Payne, M. C.; Robertson, J. Surface Diffusion: The Low Activation Energy Path for Nanotube Growth. Phys. Rev. Lett. 2005, 95, 036101.

13. Helveg, S.; Lopez-Cartes, C.; Sehested, J.; Hansen, P. L.; Clausen, B. S.; Rostrup-Nielsen, J. R.; Abild-Pedersen, F.; Nørskov, J. K. Atomic-Scale Imaging of Carbon Nanofibre Growth. Nature 2004, 427, 426-429.

14. Kodambaka, S.; Tersoff, J.; Reuter, M.; Ross, F. DiameterIndependent Kinetics in the Vapor-Liquid-Solid Growth of Si Nanowires. Phys. Rev. Lett. 2006, 96, 096105.

15. Jiang, A.; Awasthi, N.; Kolmogorov, A. N.; Setyawan, W.; Bojersson, A.; Bolton, K.; Harutyunyan, A. R.; Curtarolo, S. Theoretical Study of the Thermal Behavior of Free and Alumina-Supported Fe-C Nanoparticles. Phys. Rev. B 2007, 75, 205426.

16. Harutyunyan, A. R.; Mora, E.; Tokune, T.; Bolton, K.; Rosén, A.; Jiang, A.; Awasthi, N.; Curtarolo, S. Hidden Features of the Catalyst Nanoparticles Favorable for Single-Walled Carbon Nanotube Growth. Appl. Phys. Lett. 2007, 90, 163120.

17. Harutyunyan, A. R.; Awasthi, N.; Jiang, A.; Setyawan, W.; Mora, E.; Tokune, T.; Bolton, K.; Curtarolo, S. Reduced Carbon Solubility in Fe Nanoclusters and Implications for the Growth of Single-Walled Carbon Nanotubes. Phys. Rev. Lett. 2008, 100, 195502.

18. Amara, H.; Bichara, C.; Ducastelle, F. Understanding the Nucleation Mechanisms of Carbon Nanotubes in Catalytic Chemical Vapor Deposition. Phys. Rev. Lett. 2008, 100, 056105.

19. Baletto, F.; Ferrando, R. Structural Properties of Nanoclusters: Energetic, Thermodynamic, and Kinetic Effects. Rev. Mod. Phys. 2005, 77, 371-423.

20. Balbuena, P.; Zhao, J.; Huang, S.; Wang, Y.; Sakulchaicharoen, N.; Resasco, D. Role of the Catalyst in the Growth of Single-Wall Carbon Nanotubes. J. Nanosci. Nanotechnol. 2006, 6, 1247-1258.

21. Lyalin, A.; Hussien, A.; Solov'yov, A.; Greiner, W. Impurity Effect on the Melting of Nickel Clusters as seen via Molecular Dynamics Simulations. Phys. Rev. B 2009, 79, 165403.

22. Schebarchov, D.; Hendy, S. C. Solid-Liquid Phase Coexistence and Structural Transitions in Palladium Clusters. Phys. Rev. B 2006, 73, 121402.

23. Berry, R. S.; Beck, T. L.; Davis, H. L.; Jellinek, J. Solid-Liquid Phase Behavior in Microclusters. Adv. Chem. Phys. 1988, 70, 75-138.

24. Lindemann, F. A. The Calculation of Molecular Vibration Frequencies. Phys. Z. 1910, 11, 609-612.

25. Fick, A. Ueber Diffusion. Poggendorff's Ann. Phys. 1855, 94, 59-86.

26. Protopapas, P.; Andersen, H. C.; Parlee, N. A. D. Theory of Transport in Liquid Metals. I. Calculation of Self-Diffusion Coefficients. J. Chem. Phys. 1973, 59, 15-25.

27. Yokoyama, I. Self-diffusion Coefficient and Its Relation to Properties of Liquid Metals: A Hard-Sphere Description. Phys. B 1999, 271, 230-234.

28. De Wijs, G.; Kresse, G.; Vocadlo, L.; Dobson, D.; Alfe, D.; Gillan, M.; Price, G. The Viscosity of Liquid Iron at the Physical Conditions of the Earth's Core. Nature 1998, 392, 805-807.

29. Yokoyama, I.; Arai, T. Correlation Entropy and Its Relation to Properties of Liquid Iron, Cobalt, and Nickel. J. Noncryst. Sol. 2001, 293, 806-811.

30. Buffat, P. A. Lowering of the Melting Temperature of Small Gold Crystals between $150 \AA$ and $25 \AA$ Diameter. Thin Solid Films 1976, 32, 283-286.

31. Uppenbrink, J.; Wales, D. J. Structure and Dynamics of Model Metal Clusters. J. Chem. Phys. 1993, 98, 5720-5733.
32. Alavi, S.; Thompson, D. L. Molecular Dynamics Simulations of the Melting of Aluminum Nanoparticles. J. Phys. Chem. A 2006, 110, 1518-1523.

33. Raty, J.-Y.; Gygi, F. M. C.; Galli, G. Growth of Carbon Nanotubes on Metal Nanoparticles: A Microscopic Mechanism from ab Initio Molecular Dynamics Simulations. Phys. Rev. Lett. 2005, 95, 096103.

34. Curtarolo, S.; Awasthi, N.; Setyawan, W.; Jiang, A.; Bolton, K.; Harutyunyan, A. R. Influence of Mo on the Fe:Mo:C Nanocatalyst Thermodynamics for Single-Walled Carbon Nanotube Growth. Phys. Rev. B 2008, 78, 054105.

35. Binary Alloy Phase Diagrams; Massalski, T. B., Okamoto, H., Subramanian, P. R., Kacprzak, L., Eds.; American Society for Metals: Materials Park, OH, 1990.

36. Fischer, F. D.; Waitz, T.; Vollath, D.; Simha, N. K. On the Role of Surface Energy and Surface Stress in PhaseTransforming Nanoparticles. Prog. Mater. Sci. 2008, 53, 481-527.

37. Labastie, P.; Whetten, R. L. Statistical Thermodynamics of the Cluster Solid - Liquid Transition. Phys. Rev. Lett. 1990, $65,1567-1570$.

38. Wales, D. J.; Berry, R. S. Coexistence in Finite Systems. Phys. Rev. Lett. 1994, 73, 2875-2878.

39. Landolt-Bornstein: Diffusion in Solid Metals and Alloys III; Madelung, O., Ed.; Springer: Berlin, 1998; Vol 26.

40. Li, X.; Huang, J. Molecular Dynamics Studies of the Kinetics of Phase Changes in Clusters III: Structures, Properties, and Crystal Nucleation of Iron Nanoparticle $\mathrm{Fe}_{331}$. J. Solid State Chem. 2003, 176, 234-242.

41. Ding, F.; Rosén, A.; Curtarolo, S.; Bolton, K. Modeling the Melting of Supported Clusters. Appl. Phys. Lett. 2006, 88, 133110.

42. Ismach, A.; Joselevich, E. Orthogonal Self-Assembly of Carbon Nanotube Crossbar Architectures by Simultaneous Graphoepitaxy and Field-Directed Growth. Nano. Lett. 2006, 6, 1706-1710.

43. McNicholas, T. P.; Ding, L.; Yuan, D.; Liu, J. Density Enhancement of Aligned Single-Walled Carbon Nanotube Thin Films on Quartz Substrates by Sulfur-Assisted Synthesis. Nano Lett. 2009, 9, 3646-3650.

44. Ding, L.; Tselev, A.; Wang, J.; Yuan, D.; Chu, H.; McNicholas, T. P.; Li, Y.; Liu, J. Selective Growth of Well-Aligned Semiconducting SWCNT's. Nano Lett. 2009, 9, 800-805.

45. Cheung, C. L.; Kurtz, A.; Park, H.; Lieber, C. M. DiameterControlled Synthesis of Carbon Nanotubes. J. Phys. Chem. B 2002, 106, 2429-2433.

46. Verlet, L. Computer "Experiments" on Classical Fluids. I. Thermodynamical Properties of Lennard-Jones Molecules. Phys. Rev. 1967, 159, 98-103.

47. Hoover, W. G. Canonical Dynamics: Equilibrium PhaseSpace Distributions. Phys. Rev. A 1985, 31, 1695-1697.

48. Borjesson, A.; Curtarolo, S.; Harutyunyan, A. R.; Bolton, K. Computational Study of the Thermal Behavior of Iron Clusters on a Porous Substrate. Phys. Rev. B 2008, 77, 115450.

49. Bolton, K.; Ding, F.; Borjesson, A.; Zhu, W.; Duan, H.; Rosén, A.; Harutyunyan, A. R.; Curtarolo, S. Computational Studies of Catalytic Particles for Carbon Nanotube Growth. J. Comput. Theor. Nanosci. 2009, 6, 1-15.

50. Rosato, V.; Guillope, M.; Legrand, B. Thermodynamical and Structural Properties of fcc Transition Metals Using a Simple Tight-Binding Model. Phil. Mag. A 1989, 59, 321-336.

51. Stanek, J.; Marest, G.; Jaffrezic, H.; Binczycka, H. Interactions of Iron Implants in Transition Metals. Phys. Rev. B 1995, 52, 8414-8422.

52. Ding, F.; Bolton, K.; Rosén, A. Nucleation and Growth of Single-Walled Carbon Nanotubes: A Molecular Dynamics Study. J. Phys. Chem. B 2004, 108, 17369-17377.

53. Duan, H.; Ding, F.; Rosén, A.; Harutyunyan, A. R.; Curtarolo, S.; Bolton, K. Size-Dependent Melting Mechanisms of Iron Nanoclusters. Chem. Phys. 2007, 333, 57-62.

54. Callister, W. D. Materials Science and Engineering an Introduction; John Wiley and Son Inc: New York, 2003. 
55. McQuarrie, D. A. Statistical Mechanics; Harper and Row: New York, 1976.

56. Rapaport, D. C. The Art of Molecular Dynamics Symulations; Cambridge University Press: UK, 2004.

57. Adams, J.; Stratt, R. Extensions to the Instantaneous Normal Mode Analysis of Cluster Dynamics: Diffusion Constants and the Role of Rotations in Clusters. J. Chem. Phys. 1990, 93, 1632-1640.

58. Beck, T.; II, T. M. Dynamics of Diffusion in Small Cluster Systems. J. Chem. Phys. 1990, 93, 1347-1357. 